\title{
A Method for Measuring the Creative Potential of Computer Games
}

\author{
Wilawan Inchamnan, Peta Wyeth, Daniel Johnson, and David Conroy \\ Electrical Engineering and Computer Science, Queensland University of Technology, Australia \\ $\{w$. inchamnan, dj.conroy\} @student.qut.edu.au, \\ \{peta.wyeth, dm.johnson\} @qut. edu.au,
}

\begin{abstract}
This paper describes a method for measuring the creative potential of computer games. The research approach applies a behavioral and verbal protocol to analyze the factors that influence the creative processes used by people as they play computer games from the puzzle genre. Creative potential is measured by examining task motivation and domain-relevant and creativity-relevant skills. This paper focuses on the reliability of the factors used for measurement, determining those factors that are more strongly related to creativity. The findings show that creative potential may be determined by examining the relationship between skills required and the effect of intrinsic motivation within game play activities.
\end{abstract}

Keywords: Creative potential, Computer game, Creative measurement, Task motivation, Domain-relevant skill, Creativity-relevant skill, Behavioral assessment.

\section{Introduction}

Creative thinking processes are vital in helping people solve problems [23]. Digital simulations and games may play a significant role in facilitating exploration and creative problem solving. Although there are educational computer games that have been designed to support cognitive and creative activity [5], there is limited research to date research on how to measure the potential of a computer game to facilitate creative processes.

Assessing creative potential requires a focus on how and why an individual responds to activities [17]. However, no studies have been conducted to determine the specific interactions that occur during gameplay that support creative processes. Previous research studies have not applied coherent theories of creativity assessment to the domain of computer games. To understand the creative potential of games, criteria related to creativity activity must be clearly stated and readily translated into assessment [1].

A primary goal of this study is the development of a method for measuring the creative potential of computer games. Previous research has examined computer game play in relation to personal creative traits [8]. Our research is particularly interested in creative process, rather than the creative attributes of a person (i.e. [29]) or creative 
product. The study forms part of a program of research designed to better understand the creative processes that people engage in while playing computer games. The measurement method described in this paper has been adapted from an existing theory of creative assessment [23] and its reliability for creative assessment within the computer game context is investigated. The method developed focuses on the componential framework of creativity that includes three major components: domain-relevant skills, creativity-relevant skills and task motivation [1]. To examine the creative process potential of computer games using this comprehensive assessment technique, we have adapted the existing behavior and verbal protocol developed by Ruscio et al. [23], which has been used previously for assessing a range of creative activities. The measurement method is designed to explore the relationship between task motivation, domain relevant skills and creativity relevant skills within a particular activity.

\section{Creative Potential of Computer Games}

Creativity involves problem discovery, goal identification and engagement in heuristic activity [7],[13]. Creative problem solving is constructed using a certain set of goals, paths, and available conditions [31]. It involves sensitivity to problems and a sense of curiosity, as individuals find problems, manage discrepancies and discover answers to things they do not understand [15]. The process of becoming sensitive to problems and identifying deficiencies, missing elements and gaps in knowledge has been recognized as core to creative process [29]. It involves thinkers grasping the essential features of the problem and understanding how these features relate to the final solution [32].

Divergent and convergent thinking are core elements of the creative process. Divergent thinking is important for idea generation [3], and necessary to produce many alternative solutions to the problem [14]. Creative ideas result from the novel combination of two or more ideas that have been freed from their normal links [27]. Convergent thinking as a creative process occurs in the idea validation stage [3]. It allows an individual to select the correct way to approach the task at hand [28], with the ability to select a single response from a series of alternatives [9]. Based on the review of the literature, creative potential for this study is defined in terms of:

- Sensitivity to problems;

- The process of finding appropriate solutions through the exploration of multiple paths;

- Motivations of curiosity, discrepancies and gaps in knowledge to drive the creative problem solving process; and

- The process of evaluating solutions and settling on the most appropriate for the given problem space.

Computer games have contributed to the practice of enhancing problem-based learning processes. The experiences that occur in computer games may enhance creative processes [33]. Games support the development of critical thinking through visualization, experimentation and creativity [4]. Game elements may provide a problem 
solving experience as players break down tasks, engage meta-cognitive skills and think critically [30]. Games offer an opportunity to explore new ideas and actions through the diverse gameplay opportunities generated by communities of players. . Game experiences that are active and provide intrinsic motivation have great potential to support creative processes [20].

The componential model of creativity describes the ways in which we enter into stages of the creative activity. The componential framework of creativity has problem solving at its core and includes three major components: domain-relevant skills, creativity-relevant skills and task motivation [1]. As people solve problems they generate response possibilities from an array of available pathways and explore the environment to determine the best solution. Domain knowledge plays an important part in generation of an acceptable solution. Engaging in playful activities or fantasy can have a positive effect that influences the active engagement of creativity-relevant processes [3]. Creative-relevant skills influence the quality of the ideas produced and task motivation influences the quantity of ideas [1],[2],[3]. The three components are crucial characteristics of a creative process. We propose that the creative potential of computer games may be measured using these three major components.

\section{Assessing Creative Potential of Computer Games}

Designing an assessment lens for creative processes requires analysis and interpretation of exiting measures. Behavioral assessment allows for analysis of creativity from a divergent thinking and convergent thinking perspective. The measurement can be used in the identification and development of creative potential [26]. The method we consider focuses on behavioral observation and verbal protocol analysis during creative activity [23],[17]. Measurement of creative potential involves examining the relationship between tasks, from both a domain skill and creative skill perspective, and the effect of intrinsic motivation [23]. It has been used previously to measure creativity in structure building activities, collage making and poem writing. The research yielded a specification of particular task behaviors that strongly predict creativity. In addition, analysis of the verbal protocol yielded process measures that were strongly related to creativity [23].

\subsection{Task Motivation}

Task motivation accounts for motivation variables that encourage an individual's approach to a given task. This component is responsible for initiating and sustaining the creative process [1]. Research has demonstrated creativity is most likely to appear when a person is intrinsically motivated [3]. Task motivation includes two elements: traits that determine an individual's baseline attitude toward the task, and the individual's perceptions of a task and/or reasons for undertaking it in a given instance. Task motivation is specific to a particular task, influenced by baseline attitude toward the task and a person's own interest [6]. It is an important component of creative thinking, especially during the problem presentation stage and during response generation of the process. Task motivation may determine the difference between what an 
individual can do and what he/she will do [3]. It is assessed through an examination of social-environmental influences; that is the intrinsic and extrinsic factors that support autonomy, competence, control, and task involvement [3]. In a computer game context, intrinsic needs such as competence, autonomy, and relatedness are the core motivational dynamics that operate across the interactivity between the game and the player [21].

The model proposed by Ruscio et al. [23] identifies task motivation as a measure of involvement in tasks. Behaviors such as set breaking, task pace, exploration, enjoyment, and concentration are identified as the ways in which intrinsic motivation manifests itself within the creative process. We have taken these behaviors [23] and modified them for gaming environments in order to assess task motivation within computer games. The creative game potential measures, in terms of task motivation, are:

- Involvement (A1): Work on solving the problem

- Item 1: Participant works on solving problems within the game $\left(\mathrm{L}^{1}\right)$.

- Stability (A2): Refining the integrity or stability of a problem solution within the game

- Item 4: Participant can work on refining the integrity or stability of a problem solution within the game (L).

- Set breaking (A3): Manipulates materials; uses or attaches them in new ways

- Item 5: In-game objects and materials are able to be readily manipulated (L).

- Item 6: In-game objects and materials can be used in different ways (L).

- Pace (A4): Speed at which participant works on tasks/challenges; a slow to fast gradient of working rate

- Item 7: The speed at which participant is required to interact within the game progresses from a slow to fast gradient of working rate $(\mathrm{L})$.

- Planning (A5): Organizes material; establishes an idea, order to build in, steps to take

- Item 8: Planning is an important part of game play (L).

- Item 9: Participant can organize materials within the game (L).

- Item 10: Participant is able to establish ideas within the game (L).

- Item 11: Participant can plan the order of actions and steps to take within the game (L).

- Playfulness (A6): Engaging in tasks in curious manner; trying out ideas in a carefree way

- Item 12: Participant can engage in game tasks in a curious manner (L).

- Item 13: The game provides opportunities to try out ideas in a carefree way (L).

- Exploration (A7): Curious, or playful testing out of ideas

- Item 14: Curiosity during game exploration is encouraged (L).

- Item 15: The game encourages playful testing out of ideas (L).

\footnotetext{
${ }^{1}$ Within notation in the factors, (L) refers to Likert scale measures, (F) refers to frequency measures and (-) refers to reverse coded items.
} 
- Enjoyment (A8): Having a good time, finding pleasure in the task / challenge

- Item 16: The game allows players to have a good time (L).

- Item 17: Game play tasks are pleasurable (L).

- Concentration (A9): Focused on the task; not distracted

- Item 2: There is minimal distraction from solving problems in the game (L).

- Item 3: Participant becomes focused on the tasks in the game (L).

\subsection{Domain-Relevant Skills}

Domain-relevant skills form the basis from which any performance must proceed. This component incorporates factual knowledge, technical skills and special talents in a particular domain. The information, skills and talents that an individual brings to a task influence the creative preparation process. Domain-relevant skills define the set of possible responses available to a person [3]. Any problem domain consists of a unique set of rules and practices [31] and this knowledge allows people to identify various strategies for conducting information analysis. Domain-relevant skills provide the material drawn on during operations that determine problem-solving pathways. They also provide the criteria that will be used to assess the response possibilities [1], which are then synthesized to form a judgment [23]. Knowledge of a particular domain influences the evaluation process [6]. The process includes familiarity with and factual knowledge of the domain in question: facts, principles, and opinions within the problem-solving domain [23].

Domain-relevant skills determine the initial set of pathways to search for a solution and the ability to verify an acceptable solution [1]. Domain-relevant skills are driven by task motivation and task motivation, in turn, is increased by a positive environment and earlier success in tasks [1]. From a gaming perspective, domain-relevant skills can be evaluated through examining how well the player understands the game domain. Understanding of the game's goals and sub-goals at a particular point and the actions available to achieve these goals is important domain knowledge. Domainrelevant skills can be assessed through examination of factual knowledge of the domain in question and the technical skills demonstrated.

The measures identified by Ruscio et al. [23] as predictors of creativity are assuredness, difficulty (-) and exhibited uncertainty (-). These process factors have been adapted to formulate creative game potential measures in terms of domain-relevant skills in the context of computer game play. They include:

- Exhibited uncertainty (-) (B1): Self-initiated backtracks by using intentionally moves to previous locations or revisits a particular game task / challenge.

- Item 25: Participant reverses or undoes steps/actions performed in the game (F).

- Assuredness (B2): Confidence: certainty of ability to complete task; assuredness in going about the task; not doubtful, timid, or anxious. Pace and the speed at which particular task /challenge are addressed; a slow to fast gradient of playing rate. Difficulty solving problems encountered, trouble interacting with game elements.

- Item 18: The game allows participant to feel assured in going about required tasks (L).

- Item 19: Participant feels certain about his/her ability to complete tasks in the game (L). 
- Item 20: There is no doubt about what participant is required to do during the game (L).

- Item 21: Participant doesn't feel anxious or timid playing the game (L).

- Item 22: Participant encounters problems playing the game (L).

- Item 23: It was difficult to complete tasks in the game (L).

- Item 24: It was difficult to work with the objects/resources in the game (L).

- Difficulty (-) (B3): Problem with self: uncertainty, self-doubt, negative statements about ability or mood. Negative exclamations by using usually one word, can be two or three; curses or otherwise sharply negative statements.

- Item 26: Participant feels uncertain completing tasks in the game (F).

- Item 27: Participant has feelings of self-doubt while playing the game (F).

- Item 28: Participant produces negative statements about his/her ability as participants play the game (F).

- Item 29: Participant produces negative exclamations (e.g. curses) while playing the game (F).

\subsection{Creativity-Relevant Skills}

Creativity-relevant skills include cognitive style as well as the application of heuristics for the exploration of new concepts [1]. These factors influence the response generation process. Heuristic thinking is a skill that relies on a person's intellectual and emotional comfort with a situation. Differences in cognitive style result in different behaviors individuals apply when they gather and evaluate information [16]. Creativity-relevant skills act as an executive controller that influences the way in which the search for responses proceeds [1]. Creativity-relevant skills include the ability to concentrate for the long periods of time [6]. The relevant characteristics that are commonly reported as correlates of creative people include self-discipline, an ability to delay gratification, perseverance, and absence of conformity [6]. Problem solvers automatically activate areas of knowledge that are associated with the past problem solving experience and relevant knowledge [25]. This component of creative thinking includes the ability to break away for standard thinking, approaches and solutions during problem solving. Individuals can gain experience from idea generation that may inform their own strategies for creative thinking processes [3]. Creativityrelevant skills influence the quality of the ideas produced [1].

Creativity-relevant skills are measured through the specific process factors of concrete focus (-), concept identification, wide focus and striving [23]. These have been adapted for the gameplay context. The creative game potential measures, in terms of creativity-relevant skills, are:

- Wide focus (C1): Goal statements: Something that cannot be done in one step, future oriented; restatement of problem given, self-imposed goal, statement dealing with a desired final goal, etc. Irrelevant to task: Anything not related to performing the task / challenge.

- Item 33: The current problem that needs to be solved in the game requires more than one step (F).

- Item 34: The current problem in the game is future oriented (F).

- Item 35: Participant restates the problem presented by the game (F).

- Item 36: Participant is able to develop his/her own goals within the game (F).

- Item 37: Participant is performing actions not related to game tasks/goals (F). 
- Striving (C2): Difficulty: encountering problems or obstacles to completing some or all of the tasks/challenges. Transitions: Movement to new area of action; includes place holding utterance. Question how: Questioning how or what to do; what is currently being done. Repeat something: Repeats instructions, words or concepts presented in the game: Exclamations: based on positive or negative outcomes.

- Item 30: Participant encountered problems while completing tasks in the game (L).

- Item 31: Participant encountered obstacles while completing tasks in the game (L).

- Item 32: Participant questions what to do at particular stages in the game (L).

- Item 38: Participant transitions to a new topic area or action in the game (F).

- Item 39: Participant questioned how to complete tasks in the game (F).

- Item 40: Participant questions his/her current actions in the game (F).

- Item 41: Participant repeats instructions, words or concepts presented in the game (F).

- Item 42: Participant makes exclamations, as a positive or negative outburst (F).

- Concrete focus (-) (C3): Talks about task: statements of like or dislike about the task. Describes game elements: statement about texture, color, or other attributes of elements, naming game elements.

- Item 43: Participant makes statements of like or dislike about game tasks (F).

- Item 44: Participant talks about the qualities of the materials, objects or attributes of the game world (F).

- Concept identification (C4): Analogies: Description or statement containing an analogy or metaphor. Aha: Eureka-type statements; abrupt change in activity. Transitions: movement to new action; includes place holding utterances.

- Item 45: Participant describes action/tasks/goals in terms of analogies or metaphors (F).

- Item 46: Participant had eureka-type moments in the game (F).

- Item 47: Participant has an abrupt change in activity designed to help complete a task (F).

\section{$4 \quad$ Research Design}

This study assesses a method for measuring creativity. The methodological contribution of the study is the demonstration that creative behaviors and processes that occur during gameplay can be examined using behavioral observation techniques and verbal protocol analysis. Behavioral observation techniques and verbal protocol analysis may be applied to identify and quantify predictors of creativity [23].

The data gathered analyzes the relationship between the components of creativity, through the creative game potential measures outlined in section 3. Game task behaviors and verbalizations were coded to obtain empirical indices of the assessed factors: task motivation, domain-relevant skills and creativity-relevant skills. The observations constitute evidence of game activities that have a positive impact on creative potential. 


\subsection{Participants}

Seven participants ( 1 female) participated in the study. They had an average age of 22, and ages ranged from 18 to 34. Six of the participants were native English speakers. Seventy-one percent indicated that they played games daily, and $28.6 \%$ showed that they played games several times a week.

\subsection{Procedure}

Participation in the study involved being observed while playing three selected games: Portal 2, Braid and I-Fluid. Participants played approximately 45 minutes in total and completed a brief questionnaire on completion of each game. They played each game for 15 minutes. To examine the creative process, participants were video recorded while playing the games and a subset of players were asked to think aloud as they participated. Two researchers gathered data through behavioral and verbal coding techniques. Behavioral coding involves watching video and coding of the behaviors that occurred during game task performance. A video coding scheme was used to capture the type and frequency of observable behaviors and participant verbalizations. Video coding was completed for each of the participants, for each of the games, resulting in 21 data sets.

Coders practiced rating on pilot video records captured before the study was conducted. The researchers individually observed the game play of two people playing the three games used for the study. This resulted in 6 data sets that were examined using the Coefficient of Variation that compares variability. A data set which was collected consistently had a Coefficient of Variance of less than 20\%. Three items were refined to yield improvements in coding reliability.

\subsection{Materials}

Game related behavior was used to assess participants' level of creativity during various game activities on a seven-point Likert scale ranging from 1 (low) to 7 (high), and through the use of a frequency tally. Seventeen items related to task motivation (e.g., "Participant works on solving problems within the game."). The items were grouped within nine variables (see section 3.1): Involvement (A1), Stability (A2), Set breaking (A3), Pace (A4), Planning (A5), Playfulness (A6), Exploration (A7), Enjoyment (A8), and Concentration (A9). All task motivation items are assessed using 7-point Likert scales.

Twelve items were included to analyze domain-relevant skill factors (e.g., "Participant feels certain about his/her ability to complete tasks in the game"). The items were grouped into 3 variables (see section 3.2): Exhibited uncertainty (B1), Assuredness (B2), and Difficult (B3). Seven items are assessed using a 7-point Likert scales and data for five items is captured as frequency counts.

Eighteen items were designed to measure creativity-relevant skills (e.g., "Participant is able to develop his/her own goals within the game"). The items were grouped into four variables (see section 3.3): Wide focus (C1), Striving (C2), Concrete focus 
(C3), and Concept identification (C4). Only three items are assessed using a 7-point Likert scales. Fifteen items are assessed using frequency counts.

In order to make all items comparable to the other items, nine negative items (22, $23,24,25,26,27,28,29$, and 44) are reverse-scored. To make each variable contribute equally to the mean, values of frequency items are standardized [19].

\section{$5 \quad$ Results}

The analyses involved three measures: (A) task motivation factors, (B) domainrelevant skill factors and $(\mathrm{C})$ creativity-relevant skill factors. The internal reliability of all creative game potential measures was acceptable $(\alpha=.78)$. Differences across the three components being measured were examined using Cronbach's Alpha to determine internal reliability within each [11].

\subsection{Task Motivation Factors}

Cronbach's alpha for task motivation measures is .88, suggesting that A1 to A9 (17 items for task motivation) have relatively high internal consistency. Results indicate that removal of A9, item 3 would lead to a small improvement in Cronbach's alpha ( $\alpha$ $=.89)$. Correlation for item 3 was low $(\alpha=.057)$ and as a result the concentration factor included in item 3 (A9) should be reconsidered and potentially removed as a task motivation measure.

\subsection{Domain-Relevant Skill Factors}

The data shows an alpha coefficient of .79 for domain-relevant skill measures, suggesting that the 12 items have relatively high internal consistency. Item 22 (B2) and 25 (B1) have item-total correlation values of less than .2 [11] $(\alpha=-.59$ and $\alpha=-.02$ respectively). Analysis indicates that removal of these items leads to an improvement in Cronbach's alpha $(\alpha=.89)$ and a highly reliable 10 -item measure of domain relevant skills.

\subsection{Creativity-Relevant Skill Factors}

The data for creativity relevant skill factors produces an alpha coefficient of .75, suggesting that 18 items have relatively high internal consistency. The item-total correlation values for items 30, 33, 34, 36, 41, 44 are low $(\alpha<.2)$ [11]. Items 33, 34 and 36 are wide focus items (C1), 30 and 41 are striving items (C2) and item 44 relates to concrete focus (C3). The removal of these items leads to an improvement in Cronbach's alpha to .81. This results in a highly reliable 12-item measurement of creativity-relevant skills. 


\section{Discussion}

This paper examines a method for measurement the creative potential of puzzle-based computer games. Analysis of our adapted measurement items demonstrates that internal reliability within each component is satisfactory. Low item-total correlations for some items indicated areas for improvement.

The observation analysis used in this study identified good reliability for the 17 task motivation factors. However, item 3 is flagged as having low item-total correlation. This item is one of two concentration items (A9) designed to examine the level of a player's focus in the game. In the study, the item is phrased as "Participant becomes focused on the tasks in the game". This item has strong face validity as reflection player concentration within the game. Given the high initial alpha value and the minor improvement that results from removing item 3 (.88 to .89), we decided to keep this item. It is anticipated that improvement may be achieved with a slight rewording of the item to "Participant is focused on tasks in the game" to capture the present state of the player activity rather than an emerging behavior. The seventeen factors within categories A1 to A9 that we have identified as reliable measures in terms of task motivation are included in Fig 1.

The study data analysis resulted in acceptable reliability for the 12 domain relevant skill factors. The sole exhibited uncertainty (C1) factor had low item-total correlation. This item, "participant reverses or undoes steps/actions performed in the game", was designed to reflect uncertainty in participant behavior, yet it does so through an activity or path that is unavailable in many game experiences. Many of the rule structures and challenges embedded in games emerge through players not being able to change decisions or reverse actions or steps. Given this consideration, it is logical to remove this item. Other items relating to difficulty (B3) capture the concept of uncertainty in a way that is more appropriate in a gaming context (e.g. player feels uncertain completing tasks in the game). Analysis indicated that alpha value improvement would result from the removal of an assuredness (B2) item. This item, "participant encounters problems as he/she plays the game", is phrased in the reverse and is designed to demonstrate player assuredness. Once again, this item suffers in its translation to a gaming context. All games are designed around the idea of encountering problems and are underpinned by this notion of challenge. The notion of assuredness is better captured in the more specific items related to ability and issues with completing tasks (e.g. it was difficult to complete tasks in the game (-). Removal of these two items results in good reliability for measuring domain skills. The 10 factors within categories $\mathrm{B} 2$ and $\mathrm{B} 3$ that have been identified as reliable measures in terms of domainrelevant skill are included in Fig 1.

Analysis of study data revealed acceptable reliability for the 18 creativity skill factors used in the study. However, low item-total correlation was identified across six items. Three of these items related to wide focus (C1: item 33, item 34, and item 36). 


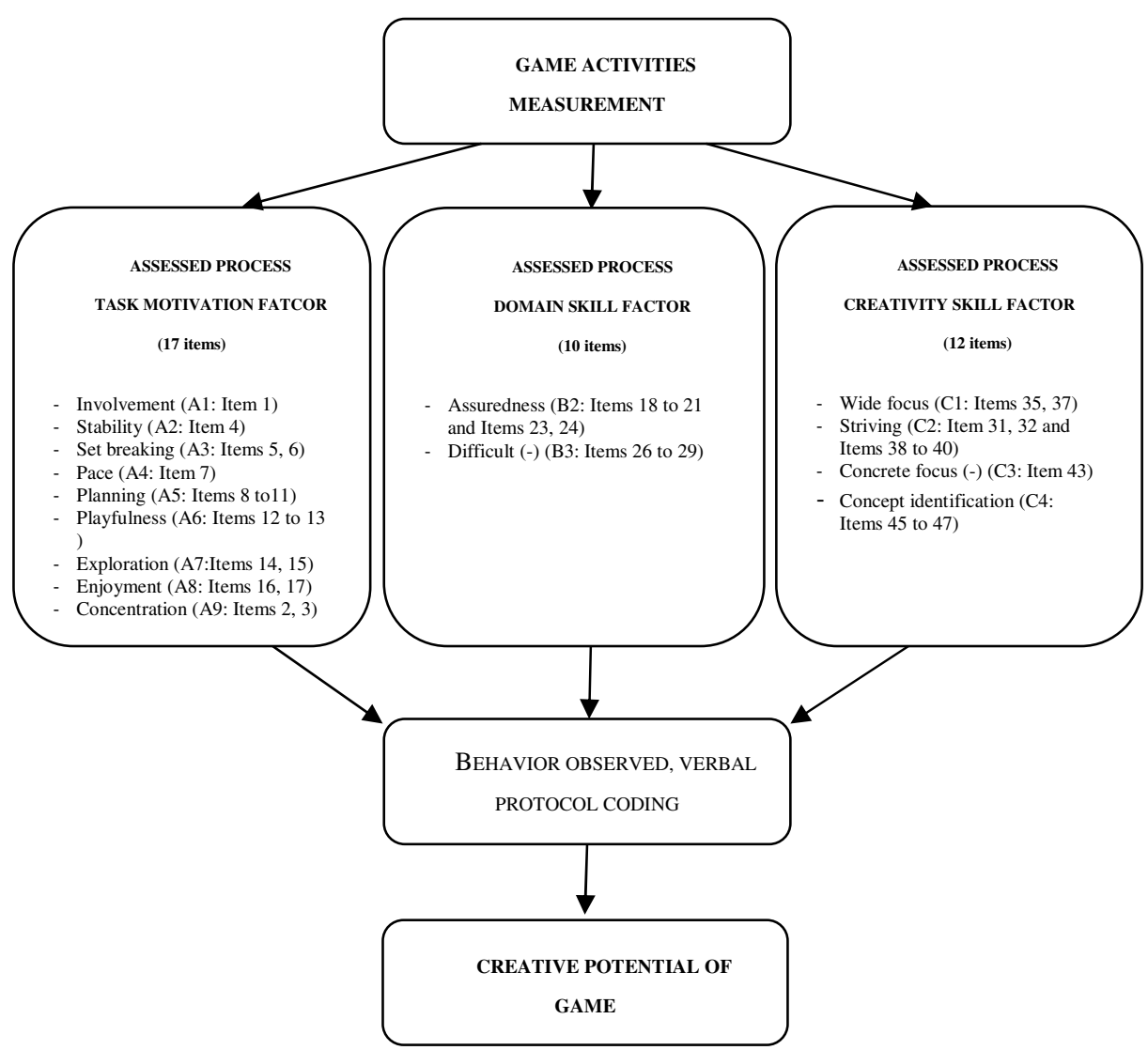

Fig. 1. A model of process factors for creative potential measurement within computer games (based on [23])

This result may be interpreted as being related to the context of gameplay generally and the puzzle game genre more specifically. Games create environments where each atomic challenge is stand-alone and is addressed that way by a player. While one problem may link to another they tend not to be interdependent. Puzzle games focus on logical and conceptual challenges and they are not generally the type of experiences where players develop their own goals. One other item that was removed to improve internal validity related to striving (C2) in terms of difficulty and problems encountered. The need to remove this item may arise from issues surrounding the notion of encountering problems discussed earlier. It may also be attributable to the items approaching the concept of striving from a difficulty point of view. Striving factors that were focused on the positive, for example achievement and questioning, had the strongest item-total correlations.

The final two items remove related to verbalization. From a concrete focus (C3) perspective, the item looked at verbalization around qualities of materials and objects in the game. This is an item that required a reversal of coded values, as creative 
processes are shown to be potentially restricted when people think about the concrete aspects of the materials they engage with [23]. We speculate that the very concrete nature of a game world, filled with interesting and perhaps unfamiliar items and objects, leads to player verbalization. The other verbalization related to striving $(\mathrm{C} 2)$ in terms of repeating instructions, words or concepts presented in the game. Within games, and puzzle games specifically, instructions are kept to a minimum and concepts encountered are centered on visual synthesis and interpretation. This might explain the improvement that would result with the removal of this item. The 12 factors within categories $\mathrm{C} 1-\mathrm{C} 4$ that have been identified as reliable measures in terms of creativity-relevant skill are included in Fig 1.

The conceptual method that was employed to assess creativity is illustrated in Fig 1. The internal reliability of the 39 items remaining as measures of creative game potential is acceptable $(\alpha=.791)$. In addition, the quality of the data and the resulting interpretations demonstrates their potential effectiveness in assessing each of the components of creativity. The creative potential prediction involves the relationship between task creativity within a knowledge domain and the effect of intrinsic motivation on the process. We have measured this creativity within a computer game context through behavior assessment and verbal protocol techniques. Data collected has resulted in a reliable measure of the creative potential of puzzle games that includes 12 items within the creativity-relevant skills factor, 10 items within the domain-relevant skills factor and 17 items within the task motivation factor.

\section{Conclusions and Future Research}

The research presented in this paper demonstrates that existing measures and techniques can be successfully adapted for use in assessing the creative processes that occur within gameplay experiences. It has identifying the key specific components of computer game experiences that may be measured to assess a game's potential for supporting creative activity. Creativity can be measured by examining domainrelevant and creativity-relevant skills as well as task motivation during game play. In future, this understanding of the ways in which games facilitate creative thinking will be used to create a framework for designing new gaming experiences. The framework will identify crucial characteristics of the creative process that emerge throughout the process of playing games and map elements of computer games to components of the creative process.

While present research has focused on the puzzle game genre and our current results cannot be extrapolated beyond this genre, future work will explore creativity in the gameplay process more generally. The knowledge generated through this research will assist in adding a new and helpful educational dimension to either educational or traditional commercial games. The design framework produced will guide game designers in the creation of games to facilitate people's creative thinking skills. 


\section{References}

1. Amabile, T.M.: The social psychology of creativity: A componential conceptualization. Journal of personality and social psychology 45, 357 (1983)

2. Amabile, T.M.: How work environments affect creativity. Paper Presented at the IEEE International Conference on Systems, Man and Cybernetics, Conference Proceedings, vol. 1, pp. 50-55. IEEE (1989)

3. Amabile, T.M.: Creativity in Context. Boulder, Colorado. Westview Press Inc. (1996)

4. Amory, A.: Game object model version II: a theoretical framework for educational game development. Educational Technology Research and Development 55, 51-77 (2007)

5. Amory, A., Seagram, R.: Educational game models: conceptualization and evaluation. South African Journal of Higher Education 17, 206-217 (2003)

6. Brown, R.T.: Creativity: What are we to measure? (1989)

7. Campbell, D.T.: Blind variation and selective retentions in creative thought as in other knowledge processes. Psychological Review 67, 380 (1960)

8. Catala, A., Jaen, J., Van Dijk, B., Jordà, S.: Exploring tabletops as an effective tool to foster creativity traits, pp. 143-150. ACM (2012)

9. Clark, C.M., Veldman, D.J., Thorpe, J.S.: Convergent and divergent thinking abilities of talented adolescents. Journal of Educational Psychology 56, 157 (1965)

10. El-Murad, J., West, D.C.: The definition and measurement of creativity: what do we know? Journal of Advertising Research 44, 188-201 (2004)

11. Everitt, B., Skrondal, A., Books24X7, I. :The Cambridge dictionary of statistics, vol. 4. Cambridge University Press, Cambridge (2002)

12. Finke, R.A., Ward, T.B., Smith, S.M.: Creative cognition: Theory, research, and applications. MIT Press, Cambridge (1992)

13. Getzels, J.W., Csikszentmihalyi, M.: The creative vision: A longitudinal study of problem finding in art. Wiley, New York (1972)

14. Gordon, W.J.J.: Synectics, the Development of Creative Capacity. Collier, New York (1961)

15. Guilford, J.P.: Creativity. American Psychologist 5, 444-454 (1950)

16. Gutierrez, O., Greenberg, E.: Creative problem solving in the specification of information requirements. Systemic Practice and Action Research 6, 647-667 (1993)

17. Kaufman, J.C., Kaufman, S.B., Lichtenberger, E.O.: Finding Creative Potential on Intelligence Tests via Divergent Production. Canadian Journal of School Psychology 26, 83-106 (2011)

18. Mumford, D., Fogarty, J., Kirwan, F.C.: Geometric invariant theory. Springer (1994)

19. Myers, J.L., Well, A.: Research design and statistical analysis, vol. 1. Lawrence Erlbaum (2003)

20. Paras, B., Bizzocchi, J.: Game, motivation, and effective learning: An integrated model for educational game design (2005)

21. Rigby, S., Ryan, R.: Glued to Games: How Video Games Draw Us in and Hold Us Spellbound. Praeger Publishers (2011)

22. Runco, M.A.: Problem finding, problem solving, and creativity. Ablex Publishing Corporation (1994)

23. Ruscio, J., Whitney, D.M., Amabile, T.M.: Looking inside the fishbowl of creativity: Verbal and behavioral predictors of creative performance. Creativity Research Journal 11, 243-263 (1998)

24. Ryan, R.M., Rigby, C.S., Przybylski, A.: The motivational pull of video games: A self-determination theory approach. Motivation and Emotion 30, 344-360 (2006) 
25. Santanen, E.L., Briggs, R.O., de Devreede, G.J.: Toward an understanding of creative solution generation, pp. 2899-2908. IEEE (2002)

26. Schaefer, C.E.: The prediction of creative achievement from a biographical inventory. Educational and Psychological Measurement 29, 431 (1969)

27. Spearman, C.E.: Creative mind. D. Appleton and company (1930)

28. Sviderskaya, N.: The EEG spatial pattern and psychophysiological characteristics of divergent and convergent thinking in humans. Human Physiology 37, 31-38 (2011)

29. Torrance, E.P.: Gifted children in the classroom. Macmillan, New York (1965)

30. Turcsányi-Szabó, M., Bedő, A., PluháR, Z.: Case study of a TeaM challenge game-ePBL revisited. Education and Information Technologies 11, 341-355 (2006)

31. Wang, Y.: On cognitive foundations of creativity and the cognitive process of creation, pp. 104-113. IEEE (2008)

32. Wertheimer, M.: Productive Thinking. University of Chicago Press, Chicago (1945)

33. Yee Leng, E., Zah Bte Wan Ali, W., Baki, R.: Computer games development experience and appreciative learning approach for creative process enhancement. Computers \& Education 55, 1131-1144 (2010) 